\title{
Introduction: Materials Research in an Aberration-Free Environment
}

The last decade has witnessed a revolution in electron microscopy as online correction of spherical aberration has become a reality in both fixed-beam and scanning instruments. The combination of improved resolution and higher beam currents coupled with the prospect of simpler image interpretation has stimulated great interest and excitement across the entire field of microscopy. The Microscopy Society of America has an active Focused Interest Group on the topic of "Materials Research in an Aberration-Free Environment," and its goal is to provide a forum for discussion and dissemination of the latest advances in instrumentation and novel applications of aberration-corrected electron microscopy. This special issue of Microscopy and Microanalysis contains contributions from the Pre-Meeting Congress on this topic held in Chicago, Illinois, in late July 2006, immediately preceding Microscopy \& Microanalysis 2006.

As described in the introductory review paper, the development of aberrationcorrected electron microscopy has had a long history. There have been many significant contributions along the path to success, and exciting new results for a variety of materials are being widely reported. However, much work still remains to be done toward fully understanding and optimizing the imaging conditions. The papers by Lentzen and Yamasaki et al. consider the closely interconnected issues of contrast, information transfer, and resolution in fixed-beam transmission electron microscopes (TEMs), while the papers by Peng et al. and Findlay et al. address similar concerns for atomic-resolution imaging with scanning mode (STEM). The benefits of combining both direct (online) and indirect (offline) approaches to aberration correction are highlighted in the article by Hetherington et al., while the paper by Geiger et al. demonstrates the extra advantages of online correction for off-axis electron holography in terms of greatly improved phase detection limits. Nellist and colleagues discuss prospects for scanning confocal microscopy using doubly-corrected (probe and imaging lenses) TEMs. Finally, several papers describe novel applications of aberration-corrected STEMs. Batson reports on the motion of gold atoms, Blom et al. describe observations on automotive emission-control catalysts, and Klie et al. report on the analysis of some complex oxides.

Overall, this special issue thus provides a contemporary snapshot of a rapidly evolving field. This type of instrumentation will continue to attract much attention in future years.

Guest Editor

David J. Smith

Arizona State University 